九州大学学術情報リポジトリ

Kyushu University Institutional Repository

\title{
A SEQUENTIAL STOCHASTIC ASSIGNMENT PROBLEM FOR A RANDOM SEQUENCE WITH UNKNOWN NUMBER OF VALUES PER PERIOD
}

Nakai, Toru

Department of Economic Engineering, Faculty of Economics, Kyushu University

Teraoka, Yoshinobu

College of Integrated Arts and Sciences, University of Osaka Prefecture

https://doi.org/10.5109/13433

出版情報: Bulletin of informatics and cybernetics. 26 (1/2)，pp.35-54，1994-03. Research Association of Statistical Sciences

バージョン :

権利関係 : 


\title{
A SEQUENTIAL STOCHASTIC ASSIGNMENT PROBLEM FOR A RANDOM SEQUENCE WITH UNKNOWN NUMBER OF VALUES PER PERIOD
}

\author{
By \\ Tōru NaKaI* and Yoshinobu Teraoka ${ }^{\dagger}$
}

\begin{abstract}
In this paper, we treat a sequential stochastic assignment problem for the random number of jobs per period. In Section 2, we consider several preliminary results about an optimal selection problem as in Nakai [10]. We treat this problem for two cases, i.e., a case with known number of arriving jobs and a one with unknown number. In Section 3, we treat a case with a previously known about the total number of arriving jobs. In Section 4, we consider a case not knowing about the number of jobs but only knowing the probability distribution of this number at each period. For these problems, there exists threshold values depending only on the distribution function of the arriving jobs. We obtain the optimal policy and the expected value obtainable by this policy by using these threshold values.
\end{abstract}

\section{Introduction}

Derman, Lieberman and Ross initially treated a sequential stochastic assignment problem in [3] at 1972. In this problem, the decision-maker hires $n$ persons, and we represent their abilities as $p_{1}, p_{2}, \cdots, p_{n}$. We assume $1 \geq p_{1} \geq p_{2} \geq \cdots \geq p_{n} \geq 0$ without loss of generality. If the decision-maker assigns a perfect man to a job with a value $x$, he will obtain a reward $x$. If he assigns a man with ability $p$, then the reward is $p x$. On the other hand, $n$ jobs will arrive in sequential order, i.e., first job 1 , next job 2 and so on. We assume to represent value of each job as a non-negative iid (independently and identically distributed) random variable with known distribution function. If a man is assigned to a job, he is unavailable for future decisions. The objective of this problem is to find an optimal policy for $n$ men which maximize the total expected reward. This policy is determined by threshold values which depend on the distribution of the jobs and independent of the $p_{1}, p_{2} \cdots, p_{n}$.

\footnotetext{
* Department of Economic Engineering, Faculty of Economics, Kyushu University, 1-19-1, Hakozaki, Higashi-Ku, Fukuoka 812, Fukuoka, Japan.

$\dagger$ College of Integrated Arts and Sciences, University of Osaka Prefecture, Gakuen 1-1, Sakai 593, Osaka, Japan.
} 
On the other hand, there is a well known property about the inequality as Lemma 1.1. (Hardy, Littlewood and Polya [4])

LEMMA 1.1. (Hardy's Lemma)

Let $a_{1} \geq a_{2} \geq \cdots \geq a_{n} \geq 0$ and $b_{1} \geq b_{2} \geq \cdots \geq b_{n} \geq 0$, then

$$
\max _{\sigma \in \mathcal{S}_{n}} \sum_{i=1}^{n} a_{i} b_{\sigma(i)}=\sum_{i=1}^{n} a_{i} b_{i},
$$

where $\mathcal{S}_{n}$ is a symmetric group on $n$ letters.

If we can observe the values of $n$ jobs at the same time, it is, therefore, optimal to assign $n$ men to $n$ jobs in sequential order. In other words, we assign the $p_{1}$ man to a job with the largest value, and $p_{2}$ to the second largest one, and so on. For the sequential stochastic assignment problem, the decision-maker could not observe $n$ jobs at the same time, and observe only one at a time. It is, therefore, a stochastic generalization of the Hardy's Lemma.

For the sequential stochastic assignment problem, there are many observations such as Albright [1] · Nakai [5] · [6] · [7] · [8] · [9] · [11] · [12] etc. In most of these sequential stochastic assignment problems, the decision-maker observes one at a time in sequential order, and not observes some of them at the same time. In this paper, we will observe a problem where the decision-maker could observe the random number of jobs at the same time.

Concerning a sequential decision problem where the number of observations at same time is a random variable, Nakai considered an optimal selection problem in [10]. For a sequence of iid random variables, the objective of this problem is to maximize the total expected reward by selecting a given number of jobs.

In Section 2, we rearrange the results obtained in Nakai [10]. In Section 2.1, we consider several preliminary results. Initially we generate a fundamental sequence and observe several properties about it. In this problem, we assume that these random variables appear uniformly and independently of the other variables. Therefore, concerning the number of observable values, it is possible to obtain the probability distribution of this number at each stage. As treated in Nakai [10], we consider an optimal selection problem in Section 2.2. By using the preliminary results, we consider the optimal policy and the total expected reward obtainable under this policy.

We also consider an optimal selection problem, where the number of arriving jobs is unknown, in Section 2.3. The only knowledge about this number is a probability distribution. We will consider similar properties in Section 2.2. We also state fundamental results as in Nakai [10]. We need the results in this section for the future observations.

In Section 3 and Section 4, we will consider a sequential stochastic assignment problem where the number of arriving jobs per period is random. If the decision-maker is able to observe only one job, this problem is similar to one considered in [3]. On the other hand, whenever the decision-maker can observe every job at the same time, this is the same to Lemma 1.1. The type of a problem treated here is, therefore, a one between these two types of problems. 
In Section 3, we treat a case with a previously known about the total number of arriving jobs. In Section 4, we consider a case not knowing about the number of jobs but only knowing the probability distribution of this number at each period. For this case, since the total number of arriving jobs is variable, it is not always impossible to assign all $n$ men to arriving jobs, but also possible to rest the jobs. This is an essential difference to the one considered in Section 3.

For these problems, there exists threshold values depending only the distribution function of the arriving jobs, which is similar to the problem such as in [3] etc. We obtain the optimal policy and the expected value obtainable by this policy by using these threshold values.

\section{Optimal Selection Problem}

\subsection{Preliminary}

Consider that $k$ jobs arrive, and let the $k$ non-negative iid random variables be $\left\{X_{i}\right\}_{i=1, \cdots, k}$ where $k=0,1, \cdots, \infty$. Let the observed values of these $k$ jobs be $x_{1}, \cdots, x_{k}$. For $x_{1}, \cdots, x_{k}$, rearrange the order from greatest to least, and let the ordered values be $\left(x_{(1)}, \cdots, x_{(k)}\right)$ where $x(1) \geq \cdots \geq x_{(k)}$. If we arrange the order from least to greatest, then the resulting values are well-known order statistics.

If $k$ jobs arrive, then the density function $g_{k, i}\left(x_{(i)}\right)$ of the $i$-th greatest value $X_{(i)}$ is given by

$$
g_{k, i}\left(x_{(i)}\right)=\frac{k !}{(i-1) !(k-1) !}\left(F\left(x_{(i)}\right)\right)^{k-i}\left(1-F\left(x_{(i)}\right)\right)^{i-1} f\left(x_{(i)}\right)\left(x_{1}, \cdots, x_{k}\right) .
$$

We assume the $X$ 's to be absolutely continuous with a density function as $f(x)$.

Consider a sequence where $0=\infty \geq a_{1} \geq \cdots \geq a_{n} \geq \cdots \geq 0$. Initially we define a non-negative function $U_{k}\left(a_{i}, a_{i-1} \mid l, y\right)$ as follows.

$$
\begin{aligned}
U_{k}\left(a_{i}, a_{i-1} \mid l, y\right) & =\int_{0}^{a_{i} \wedge y} x_{(l)} h_{k, l}\left(x_{(l)}\right) f\left(x_{(l)}\right) d x_{(l)} \\
& +\int_{a_{i-1} \wedge y}^{y} U_{k}\left(a_{i-1}, a_{i-2} \mid l+1, x_{(l)}\right) f\left(x_{(l)}\right) d x_{(l)}
\end{aligned}
$$

where

$$
U_{k}\left(a_{i}, a_{i-1} \mid k+1, y\right)=a_{i} \quad(y \geq 0)
$$

and

$$
h_{k, l}\left(x_{(l)}\right)=\frac{k !}{(k-l) !}\left(F\left(x_{(l)}\right)\right)^{k-l} .
$$

Next we construct a sequence $\left\{\alpha_{i, k}\right\}_{i=0,1, \ldots}$ of non-negative numbers as follows.

$$
\alpha_{i, k}=U_{k}\left(a_{i}, a_{i-1} \mid 1, \infty\right) \quad(i=1,2, \cdots),
$$

where $\alpha_{0, k}=\infty$. For this sequence, we obtain Lemmas 2.1, 2.3, 2.4 and 2.5. For the proofs of these properties, see Nakai [10]. 
REMARK 2.1. Concerning a function $U_{k}\left(a_{i}, a_{i-1} \mid l, y\right)$ defined in (2.2), we remark the next things.

$$
\begin{aligned}
\alpha_{1, k}= & U_{k}\left(a_{i}, a_{i-1} \mid 1, \infty\right) \\
= & \int_{0}^{a_{i}} a_{i} h_{k, 1}\left(x_{(1)}\right) f\left(x_{(1)}\right) d x_{(1)}+\int_{a_{i}}^{a_{i-1}} x_{(1)} h_{k, 1}\left(x_{(1)}\right) f\left(x_{(1)}\right) d x_{(1)} \\
& +\int_{a_{i-1}}^{y} U_{k}\left(a_{i-1}, a_{i-2} \mid 2, x_{(1)}\right) f\left(x_{(1)}\right) d x_{(1)}
\end{aligned}
$$

and

$$
\begin{aligned}
\alpha_{1, k} & =U_{k}\left(a_{1}, \infty \mid 1, \infty\right) \\
& =\int_{0}^{a_{1}} a_{1} h_{k, 1}\left(x_{(1)}\right) f\left(x_{(1)}\right) d x_{(1)}+\int_{a_{1}}^{\infty} x_{(1)} h_{k, 1}\left(x_{(1)}\right) f\left(x_{(1)}\right) d x_{(1)}
\end{aligned}
$$

Lemma 2.1. For a sequence $\left\{\alpha_{i, k}\right\}_{i=0,1, \ldots}$, it has a following property.

$$
\alpha_{1, k} \geq \alpha_{2, k} \geq \cdots \geq \alpha_{i, k} \geq 0
$$

In other words, it is also a non-increasing sequence with respect to $i$.

Lemma 2.2. For two sequences $\left\{a_{i}\right\}_{i=0,1, \ldots}$ and $\left\{\alpha_{i, k}\right\}_{i=0,1, \ldots,} \alpha_{i, k} \geq a_{i}$.

LEMMA 2.3. The sequence $\left\{\alpha_{i, k}\right\}_{i=0,1, \ldots}$ is obtained by the following equations.

$$
\begin{aligned}
\alpha_{i, k} & =\sum_{j=1}^{k \wedge i} \int_{a_{i-j+1}}^{a_{i-j}} x_{(j)} g_{k, j}\left(x_{(j)}\right) d x_{(j)} \\
& +\sum_{j=0}^{k \wedge(i-1)} a_{i-j k} C_{j}\left(1-F\left(a_{i-j}\right)\right)^{j}\left(F\left(a_{i-j}\right)\right)^{k-j}
\end{aligned}
$$

LEMMA 2.4. For two sequences $\left\{a_{i}\right\}_{i=0,1, \cdots}$ and $\left\{\alpha_{i, k}\right\}_{i=0,1, \cdots}$

$$
\alpha_{i, k}=\mathrm{E}\left[X_{(i)}\right],(1 \leq i \leq k) \quad \alpha_{i, k}=0(i>k)
$$

LEMMA 2.5. Let $\left\{a_{i}\right\}_{i=1,2, \ldots}$ and $\left\{b_{i}\right\}_{i=1,2, \cdots}$ be two decreasing sequences of nonnegative numbers. If $a_{i}>b_{i}(i=1,2, \cdots)$, then the function $U_{k}\left(a_{i}, a_{i-1} \mid l, y\right)$ has the following property.

$$
U_{k}\left(a_{i}, a_{i-1} \mid l, y\right)-U_{k}\left(b_{i}, b_{i-1} \mid l, y\right) \geq 0
$$




\subsection{Optimal Selection Problem (The Case of Known Number of Jobs)}

We consider an optimal selection problem which is a generalization of a well-known secretary problem. We describe a basic problem as follows. Suppose an employer wants to employ $n$ jobs within the next $N$ periods, and there are $m(\geq n)$ jobs. His objective is to maximize the total expected reward of the jobs. Each period, the employer observes several of these jobs. The number of jobs on a given period is a random variable, and the employer knows the probability distribution of this number on any given day. When the number of jobs on a given day is $k$, the employer observes the amounts of these $k$ jobs. After that, he decides whether to select or to reject for each job (each job will accept an offer). The number of remaining jobs becomes $m-n$, since we treat a problem without recall. We assume an amount of each job as iid random variable $\left\{X_{i}\right\}_{i=1, \cdots, m}$.

During $N$ periods, each job chooses an appearing period at random, independently of the other jobs. Consider that $N$ periods remain. Then the probability of appearing on the initial period is $\frac{1}{N}$ for each job. The number of observed jobs is, therefore, not always one, but also several. The unselected job is not available for future decisions. Nakai treated in [10], and omit the proofs for this case.

Consider that $N$ periods remain, and there are $m$ jobs for $n$ positions $(n \leq m)$. We consider $(N, m, n)$ be the state variable, and refer to this problem as $P_{N, m, n}$. For this problem, if the number of arriving jobs on the initial period is $k$, we refer this sub-problem as $P_{N, m, n}(k)$. When the ordered values of the amounts of $k$ jobs are $x_{(1)}, \cdots, x_{(k)}$, we refer to this sub-problem as $P_{N, m, n}\left(k ; x_{(1)}, \cdots, x_{(k)}\right)$.

In general, when the problem is in state $(N, m, n)$, let $p_{N, m}(k)(k=0,1, \cdots, m)$ be a probability that $k$ jobs arrive on the initial period. Since each job chooses an arriving period at random, this probabilities $\left\{p_{N, m}(k)\right\}_{k=0,1, \cdots, m}$ is equal to

$$
p_{N, m}(k)={ }_{m} C_{k} \frac{(N-1)^{m-k}}{N^{m}} . \quad\left(0 \leq k \leq m, p_{1, m}(m)=1\right)
$$

For the general case, we can obtain similar results for the general case.

The objective of this problem is to maximize the total expected amounts from the $n$ selected jobs. We will obtain the optimal policy for this problem and get the total expected amounts obtainable under this policy. We also consider the properties about problem.

Moreover, let $v_{N, m, n}, v_{N, m, n}(k)$ and $v_{N, m, n}\left(k ; x_{(1)}, \cdots, x_{(k)}\right)$ be the total expected amounts in an optimal policy for $P_{N, m, n}, P_{N, m, n}(k)$ and $P_{N, m, n}\left(k ; x_{(1)}, \cdots, x_{(k)}\right)$, respectively. These values exist and satisfy the recursive equations in Equations (2.9) to (2.11).

$$
\begin{aligned}
v_{N, m, n} & =\sum_{k=0}^{m} v_{N, m, n}(k) p_{N, m}(k) \\
v_{N, m, n}(k) & =E\left[v_{N, m, n}\left(k ; X_{(1)}, \cdots, X_{(k)}\right)\right] \\
v_{N, m, n}\left(k ; x_{(1)}, \cdots, x_{(k)}\right) & =\max _{1 \leq i \leq k}\left\{\sum_{j=1}^{i} x_{(j)}+v_{N-1, m-k, n-i}\right\}
\end{aligned}
$$


We can derive these equations from the dynamic programming formulation of $P_{N, m, n}$. Concerning Equation (2.11), we note that the following. If the employer decides to select $i$ jobs out of $k(i \leq k)$, he will select the $i$ jobs with the largest values. Hence, we should first consider the job with the largest value. If the employer rejects these jobs, then he rejects the others observed on that period as well. If the employer selects the first job, the employer decides whether to select or reject a job with the second largest value, and so on. Thus, in order to achieve his objective, the employer makes decisions regarding the jobs in the order of their magnitude of amounts.

We next define two sequences $\left\{a_{N, m}^{i}\right\}_{i=1, \cdots}$ and $\left\{a_{N, m}^{i}(k)\right\}_{i=1, \cdots}$ of non-negative numbers. We recursively determine this sequence as a function of $N$ in the following manner where $0 \leq k \leq m$.

$$
\begin{aligned}
a_{N, m}^{i} & =\sum_{k=0}^{m} a_{N, m}^{i}(k) p_{N, m}(k), \\
a_{N, m}^{i}(k) & =U_{k}\left(a_{N-1, m-k}^{i}, a_{N-1, m-k}^{i-1} \mid 1, \infty\right), \\
a_{N, m}^{i}(0) & =a_{N-1, m}^{i},
\end{aligned}
$$

where $a_{N, m}^{0}=a_{N, m}^{0}(k)=\infty, a_{0,0}^{i}=0$. We call these values as the threshold values of this problem.

We get the optimal policy for this problem and the total expected value obtainable under this policy in the next two propositions. (See the details in Nakai [10])

Proposition 2.1. When the problem is in state $(N, m, n)$, supposes that the employer observes $k$ jobs with ordered values $\left(x_{(1)}, \cdots, x_{(k)}\right)$. The optimal policy for the employer is as follows.

Let $j$ be the largest number that satisfies $x_{(j)} \geq a_{N-1, m-k}^{k-j+1}$ and $1 \leq j \leq k \wedge n$, i.e., $x_{(j+1)}<a_{N-1, m-k}^{k-j}$ or $j=k \wedge n$. Then the employer decides to select $j$ jobs with the largest value, i.e., $x_{(1)}, \cdots, x_{(j)}$. If no such $j$ exists, the decision-maker selects no job.

PROPOSITION 2.2. The values $v_{N, m, n}$ and $v_{N, m, n}(k)$ satisfy the following equations.

$$
\begin{aligned}
v_{N, m, n} & =\sum_{i=1}^{n} a_{N, m}^{i}, \\
v_{N, m, n}(k) & =\sum_{i=1}^{k} a_{k, m}^{i}(k) .
\end{aligned}
$$

Lemma 2.3 yields the next proposition.

Proposition 2.3. The sequences $\left\{a_{N, m}^{i}\right\}_{i=1,2, \ldots}$ and $\left\{a_{N, m}^{i}(k)\right\}_{i=1,2, \ldots}$ satisfy the following recursive equations, for $n \geq 1$.

$$
a_{N, m}^{i}(k)=\sum_{j=1}^{m \wedge i} \int_{a_{N-1, m-k}^{i-j+1}}^{a_{N-1, m-k}^{i-j}} x_{(j)} g_{M, j}\left(x_{(j)}\right) d x_{(j)}
$$




$$
\begin{aligned}
+ & \sum_{j=0}^{k \wedge(i-1)} a_{N-1, m-k}^{i-j} C_{j}\left(1-F\left(a_{N-1, m-k}^{i-j}\right)\right)^{j}\left(F\left(a_{N-1, m-k}^{i-j}\right)\right)^{m-j}, \\
a_{N, m}^{i}= & \sum_{m=0}^{m} a_{N, m}^{i}(k) p_{N, m}(k)
\end{aligned}
$$

From Lemma 2.4, we get the following relations.

$$
\begin{aligned}
a_{1, m}^{i} & =a_{1, m}^{i}(m), \\
a_{1, m}^{i}(m) & =\mathrm{E}\left[X_{(i)}\right] .
\end{aligned}
$$

Lemma 2.1 yields Corollary 2.1.

Corollary 2.1. The two sequences $\left\{a_{N, m}^{i}\right\}_{i=1,2, \ldots}$ and $\left\{a_{N, m}^{i}(k)\right\}_{i=1,2, \ldots}$ are non-increasing sequences with respect to $i$.

REMARK 2.2. By this corollary, the sequence $\left\{a_{N, m}^{k}\right\}_{k=1,2, \ldots}$ is a non-increasing sequence with respect to $k$. However $v_{N, m, n}$ and $v_{N, m, n}(k)$ are not always increasing or decreasing with respect to $N$. We can not show these facts. This situation arises because the decision-maker knows the total number of jobs a priori.

\subsection{An Optimal Selection Problem with Unknown Number of jobs}

We consider an optimal selection problem where the total number of jobs is unknown a priori. We also assume a same situation as considered in the last subsection. During given planning period, each job appears to the decision-maker with an amount which is a realization of the iid random variables $\left\{X_{i}\right\}_{i=1, \cdots, \infty}$. When $N$ periods remain, let $p_{N}(k)(k=0,1, \cdots, \infty)$ be a probability that $k$ jobs appear at the initial period. We also assume that $\left(\sum_{k=1}^{\infty} k p_{N}(k)<\infty\right)$ and the random variables $\left\{X_{i}\right\}_{i=1, \cdots, \infty}$ are absolutely continuous with a density function $f(x)$.

During $N$ time period, the employer observes the arriving jobs, and selects $n$ of them in order to maximize the total expected amount. We consider $(N, n)$ as a state variable of this problem, and let $Q_{N, n}$ be a problem in this state. For the optimal selection problem $Q(N, n)$, let $Q_{N, n}(k)$ be a sub-problem of this problem when the employer observes $k$ jobs. When the ordered values of these $k$ jobs are $x_{(1)}, \cdots, x_{(k)}$, let $Q_{N, n}\left(k ; x_{(1)}, \cdots, x_{(k)}\right)$ be a sub-problem for this situation.

For the problems $Q_{N, n}, Q_{N, n}(k)$ and $Q_{N, n}\left(k ; x_{(1)}, \cdots, x_{(k)}\right)$, let $v_{N, n}^{*}, v_{N, n}^{*}(k)$ and $v_{N, n}^{*}\left(k ; x_{(1)}, \cdots, x_{(k)}\right)$ be total expected reward obtainable under the optimal policy, respectively. By the optimality principle, we obtain the following recursive equations.

$$
\begin{aligned}
v_{N, n}^{*} & =\sum_{k=0}^{\infty} v_{N, n}^{*}(k) p_{N}(k) \\
v_{N, n}^{*}(k) & =E\left[v_{N, n}^{*}\left(k ; X_{(1)}, \cdots, X_{(k)}\right)\right] \\
v_{N, n}^{*}\left(k ; x_{(1)}, \cdots, x_{(k)}\right) & =\max _{1 \leq i \leq k}\left\{\sum_{j=1}^{i} x_{(j)}+v_{N-1, n-i}^{*}\right\} .
\end{aligned}
$$


Concerning these recursive equations, we note similar comments as in the last subsection.

Recursively define two sequences, $\left\{a_{N}^{i}\right\}_{i=1, \cdots}$ and $\left\{a_{N}^{i}(k)\right\}_{i=1, \cdots}$ of non-negative numbers, where $0 \leq k \leq \infty$.

$$
\begin{aligned}
a_{N}^{i} & =\sum_{k=0}^{\infty} a_{N}^{i}(k) p_{N}(k), \\
a_{N}^{i}(k) & =U_{k}\left(a_{N-1}^{i}, a_{N-1}^{i-1} \mid 1, \infty\right), \\
a_{N}^{i}(0) & =a_{N-1}^{i},
\end{aligned}
$$

where

$$
a_{N}^{0}=a_{N}^{0}(k)=\infty, \quad a_{0}^{i}=0 .
$$

REMARK 2.3. For the problem in the last sub-section, we defined that

$$
a_{1, k}^{i}=a_{1, k}^{i}(k)=U_{k}(0, \infty \mid 1, \infty)=\mathrm{E}\left[X_{(k)}\right] .
$$

For the problem considered here, we define $a_{1}^{i}$ and $a_{1}^{i}(k)$ as follows.

$$
\begin{aligned}
a_{1}^{i} & =\sum_{k=0}^{\infty} a_{1}^{i}(k) p_{1}(k), \\
a_{1}^{i}(k) & =U_{k}(0, \infty \mid 1, \infty)=\mathrm{E}\left[X_{(i)}^{k}\right] . \quad(1 \leq i<k)
\end{aligned}
$$

We consider that $X_{(i)}^{k}$ is the $i$-th largest value for the $k$ iid random variables.

We have already obtained the solution for this optimal selection problem as the next propositions. (Nakai [10])

Proposition 2.4. The optimal policy of an optimal selection problem $Q_{N, n}$ is stated as follows.

When the problem is in state $(N, n)$, we suppose a condition that the employer observes $k$ jobs with ordered value $\left(x_{(1)}, \cdots, x_{(k)}\right)$. The optimal policy for the employer is as follows.

Let $j$ be the largest number that satisfies $x_{(j)} \geq a_{N-1}^{k-j+1}$ and $1 \leq j \leq k \wedge n$, i.e., $x_{(j+1)}<a_{N-1}^{k-j}$ or $j=k \wedge n$. Then the employer decides to select $j$ jobs with the largest value, i.e., $x_{(1)}, \cdots, x_{(j)}$. If no such $j$ exists, the decision-maker selects no job.

Proposition 2.5. For the problems $Q_{N, n}$ and $Q_{N, n}(k)$, the values of $v_{N, n}^{*}$ and $v_{N, n}^{*}(k)$ satisfy the following equations.

$$
\begin{aligned}
v_{N, n}^{*} & =\sum_{i=1}^{n} a_{N}^{i}, \\
v_{N, n}^{*}(k) & =\sum_{i=1}^{k} a_{k}^{i}(k) .
\end{aligned}
$$


We have already obtained these two propositions in Nakai [10], and omit the proofs here.

Lemma 2.3 yields the next proposition.

Proposition 2.6. The two sequences $\left\{a_{N}^{i}\right\}_{i=1,2, \ldots}$ and $\left\{a_{N}^{i}(k)\right\}_{i=1,2, \ldots}$ of nonnegative numbers satisfy the following relations.

$$
\begin{aligned}
& a_{N}^{i}(k)=\sum_{j=1}^{i} \int_{a_{N-1}^{i-j+1}}^{a_{N-1}^{i-j}} x_{(j)} g_{M, j}\left(x_{(j)}\right) d x_{(j)} \\
& +\sum_{j=0}^{k \wedge(i-1)} a_{N-1 k}^{i-j} C_{j}\left(1-F\left(a_{N-1}^{i-j}\right)\right)^{j}\left(F\left(a_{N-1}^{i-j}\right)\right)^{i-j} \\
& a_{N}^{i}=\sum_{m=0}^{\infty} a_{N}^{i}(k) p_{N}(k)
\end{aligned}
$$

By Lemma 2.4, we obtain the equations

$$
\begin{aligned}
a_{1}^{i} & =\sum_{k=0}^{\infty} a_{1}^{i}(k) p_{k}, \\
a_{1}^{i}(k) & =\mathrm{E}\left[X_{(i)}\right] .
\end{aligned}
$$

Lemma 2.1 yields the next property. to $i$.

CoRollary 2.2. $\left\{a_{N}^{i}\right\}_{i=1,2, \ldots}$ and $\left\{a_{N}^{i}(k)\right\}_{i=1,2, \ldots}$ are non-increasing with respect

By Proposition 2.5, we get the next lemma obviously.

LEMMA 2.6. $v_{N, n}^{*}(k)$ and $v_{N, n}^{*}$ are increasing with respect to $n$.

LEMMA 2.7. $\left\{a_{N}^{i}\right\}_{i=1,2, \ldots}$ and $\left\{a_{N}^{i}(k)\right\}_{i=1,2, \cdots}(1 \leq k \leq n)$ are non-decreasing sequences with respect to $N$.

Proof. We employ the induction principle on $N$. It is clear for the case $N=1$. We assume this lemma for any values less than $N-1$. Next we are going to prove this lemma for the case with $N$.

Since

Lemma 2.2 yields

$$
a_{N}^{i}(k)=U_{k}\left(a_{N-1}^{i}, a_{N-1}^{i-1} \mid 1, \infty\right)
$$

$$
a_{N}^{i}(k) \geq a_{N-1}^{i}
$$

We get, therefore, the following inequality.

$$
\begin{aligned}
a_{N}^{i} & \geq \sum_{k=0}^{\infty} a_{N-1}^{i} p_{N}(k) \\
& =a_{N-1}^{i} \sum_{k=0}^{\infty} p_{N}(k) \\
& =a_{N-1}^{i},
\end{aligned}
$$


since

$$
a_{N}^{i}=\sum_{k=0}^{\infty} a_{N}^{i}(k) p_{N}(k)
$$

This completes the proof.

REMARK 2.4. For the problem in the last subsection, we can not obtain a similar inequality

$$
a_{N, m}^{i} \geq \sum_{k=0}^{\infty} a_{N-1, m-k}^{i} p_{N}(k) .
$$

It is, therefore, difficult to show the property similar to this lemma.

LEMMA 2.8. $v_{N, n}^{*}$ and $v_{N, n}^{*}(k)$ are non-decreasing functions with respect to $N$.

Proof. Since

$$
v_{N, n}^{*}=\sum_{i=1}^{n} a_{N}^{i} \quad \text { and } \quad v_{N, n}^{*}(k)=\sum_{i=1}^{k} a_{k}^{i}(k),
$$

Lemma 2.7 yields this lemma.

\section{Sequential Stochastic Assignment Problem with Known Number of jobs}

Concerning an optimal selection problem, we consider a sequential stochastic assignment problem. In this problem, the decision-maker hires $n$ persons with abilities as $p_{1}, p_{2}, \cdots, p_{n}$ where $1 \geq p_{1} \geq p_{2} \geq \cdots \geq p_{n} \geq 0$. If he assigns a perfect man to a job with a value $x$, he will obtain a reward $x$. If he assigns a man with ability $p$, then the reward is $p x$. On the other hand, $m$ jobs will arrive in sequential order, i.e., first job 1 , next job 2 and so on, but the number of observable jobs at one time is not only one. We consider a value of each job as iid random variable with known distribution function. Each period, the employer observes several of these jobs. The number of jobs on a given period is a random variable, and the employer knows the probability distribution of this number on any period. When the number of jobs on a given period is $k$, the employer observes the amounts of these $k$ jobs. If a man is assigned to a job, he is unavailable for future decisions. The objective of this problem is to find an optimal policy which maximizes the total expected reward. This policy is determined by threshold values which depend on the distribution function of the jobs and independent of the $p_{1}, p_{2} \cdots, p_{n}$.

In this section, we will consider the above problem for the case that the decisionmaker knows previously the total number of jobs. This is a version of the optimal selection problem treated in 2.2 .

Concerning this problem, there exists a sequence of threshold values which is independent of $p_{1}, p_{2}, \cdots, p_{n}$ but depends on the distribution function of the random variables. These values determine the optimal policy and the value obtainable under this policy. This result is similar to one that is obtained for sequential stochastic assignment problems treated before. 
Since the objective of this problem is to maximize the total expected reward, if $m<n$, then we only consider the $m$ largest $p$ 's from $\left\{p_{1}, \cdots, p_{n}\right\}$, i.e., $\left\{p_{1}, \cdots, p_{m}\right\}$. On the other hand, if $m>n$, then we add $m-n$ persons with abilities $p_{n+1}=\cdots=p_{m}=0$. We assume, therefore, $m=n$ without loss of generality, and we omit the parameter $n$ in this section.

Consider that $N$ periods remain, and there are $m$ jobs for $m$ positions with abilities, $p_{1}, \cdots, p_{m}$. We consider the $\left(N ; p_{1}, \cdots, p_{m}\right)$ as a state variable of this problem, and refer this problem as $P_{N}\left(p_{1}, \cdots, p_{m}\right)$. For this problem, if the number of jobs on the initial period is $k$, we refer this sub-problem as $P_{N ; p_{1}, \cdots, p_{m}}(k)$. When the ordered values of these $k$ jobs are $x_{(1)}, \cdots, x_{(k)}$, we refer this sub-problem as $P_{N ; p_{1}, \cdots, p_{m}}\left(k ; x_{(1)}, \cdots, x_{(k)}\right)$.

Let $v_{N ; p_{1}, \cdots, p_{m}}$ be the total expected reward obtainable under the optimal policy for the problem $P_{N_{;} p_{1}, \cdots, p_{m}}$. Let $v_{N ; p_{1}, \cdots, p_{m}}(k)$ be the one for $P_{N_{; p_{1}}, \cdots, p_{m}}(k)$. Let $v_{N ; p_{1}, \cdots, p_{m}}\left(k ; x_{(1)}, \cdots, x_{(k)}\right)$ be the one for $P_{N ; p_{1}, \cdots, p_{m}}\left(k ; x_{(1)}, \cdots, x_{(k)}\right)$. By the optimality principle (see Ross [13]), we get the following recursive equations.

$$
\begin{aligned}
& v_{N ; p_{1}, \cdots, p_{m}}=\sum_{k=0}^{m} v_{N ; p_{1}, \cdots, p_{m}}(k) p_{N}(k), \\
& v_{N ; p_{1}, \cdots, p_{m}}(k)=E\left[v_{N ; p_{1}, \cdots, p_{m}}\left(k ; X_{(1)}, \cdots, X_{(k)}\right)\right], \\
& v_{N ; p_{1}, \cdots, p_{m}}\left(k ; x_{(1)}, \cdots, x_{(k)}\right)= \\
& \max _{\left\{\bar{p}_{1}, \cdots, \bar{p}_{k}\right\} \subset\left\{p_{1}, \cdots, p_{m}\right\}} \max _{\sigma \in \mathcal{S}_{k}}\left\{\sum_{j=1}^{k} \bar{p}_{\sigma(j)} x_{(j)}+v_{N-1 ; p_{1}^{*}, \cdots, p_{m-k}^{*}}\right\} .
\end{aligned}
$$

Let $\left\{p_{1}^{*}, \cdots, p_{m-k}^{*}\right\}$ be a set of $\left\{p_{1}, \cdots, p_{m}\right\}-\left\{\bar{p}_{1}, \cdots, \bar{p}_{k}\right\}$ where $p_{1}^{*} \geq \cdots \geq p_{m-k}^{*}$ and $\bar{p}_{1} \geq \cdots \geq \bar{p}_{k}$.

Proposition 3.1. The optimal policy for the sequential stochastic assignment problem $P_{N ; p_{1}, \cdots, p_{m}}$ can be described as follows.

Let the state of this problem be $\left(N ; p_{1}, \cdots, p_{m}\right)$, and let the ordered values of $k$ arriving jobs be $x_{(1)}, \cdots, x_{(k)}$. For the union of two sets, $\left\{x_{(i)}\right\}_{i=1, \cdots, k}$ and $\left\{a_{N-1, m-k}^{i}\right\}_{i=1, \cdots, m-k}$, we rearrange the order from largest to least. Let this set be $\left\{b_{j}\right\}_{j=1,2, \cdots, m}$. If $b_{j}=x_{(i)}$ for $j=1, \cdots, m$ and $i=1, \cdots, k$, then it is optimal to assign the job with a value $x_{(i)}$ to the $j$-th $p_{j}$. If $b_{j}=a_{N-1, m-k}^{i}$ for $j=1, \cdots, m$ and $i=1, \cdots, m-k$, then it is optimal not to assign at this period to the $j$-th $p_{j}$.

PRoposition 3.2. The total expected reward $v_{N ; p_{1}, \cdots, p_{m}}$ and $v_{N ; p_{1}, \cdots, p_{m}}(k)$ obtainable under the optimal policy are obtained as follows.

$$
\begin{aligned}
v_{N ; p_{1}, \cdots, p_{m}} & =\sum_{i=1}^{m} p_{i} a_{N, m}^{i} \\
v_{N ; p_{1}, \cdots, p_{m}}(k) & =\sum_{i=1}^{m} p_{i} a_{N, m}^{i}(k) .
\end{aligned}
$$


In order to prove these two propositions, we employ the induction principle on $N$. We will prove Proposition 3.1 and Proposition 3.2 in the same time. First we prove these propositions for $N=1$. By assuming these propositions for any values less than $N-1$, we will prove Proposition 3.1 and Proposition 3.2 for $N$.

When $N=1$, we have

$$
\max _{\sigma \in \mathcal{S}_{k}}\left\{\sum_{j=1}^{k} \bar{p}_{\sigma(j)} x_{(j)}\right\} .
$$

Lemma 1.1 yields Proposition 3.1. Since

$$
\begin{aligned}
v_{N ; p_{1}, \cdots, p_{m}} & =\sum_{i=1}^{m} p_{i} E\left[X_{(i)}\right] \\
& =\sum_{i=1}^{m} p_{i} a_{N, m}^{i}
\end{aligned}
$$

we get Proposition 3.2. We assume these propositions for any value less than $N-1$, and then we prove these propositions for $N$.

Proof of Proposition 3.1. Initially consider the following fact.

$$
\max _{\sigma \in \mathcal{S}_{k}}\left\{\sum_{j=1}^{k} \bar{p}_{\sigma(j)} x_{(j)}+v_{N-1 ; p_{1}^{*}, \cdots, p_{m-k}^{*}}\right\}
$$

By the induction assumption,

$$
v_{N-1 ; p_{1}^{*}, \cdots, p_{m-k}^{*}}=\sum_{i=1}^{m-k} p_{i}^{*} a_{N-1, m-k}^{i}
$$

This yields, therefore,

$$
\begin{aligned}
\max _{\sigma \in \mathcal{S}_{k}}\left\{\sum_{j=1}^{k} \bar{p}_{\sigma(j)} x_{(j)}+v_{N-1 ; p_{1}^{*}, \cdots, p_{m-k}^{*}}\right\} & =\max _{\sigma \in \mathcal{S}_{k}}\left\{\sum_{j=1}^{k} \bar{p}_{\sigma(j)} x_{(j)}+\sum_{i=1}^{m-k} p_{i}^{*} a_{N-1, m-k}^{i}\right\} \\
& =\max _{\sigma \in \mathcal{S}_{k}}\left\{\sum_{j=1}^{k} \bar{p}_{\sigma(j)} x_{(j)}\right\}+\sum_{i=1}^{m-k} p_{i}^{*} a_{N-1, m-k}^{i}
\end{aligned}
$$

Lemma 1.1 and Equations (3.6) yield

$$
\max _{\sigma \in \mathcal{S}_{k}}\left\{\sum_{j=1}^{k} \bar{p}_{\sigma(j)} x_{(j)}\right\}+\sum_{i=1}^{m-k} p_{i}^{*} a_{N-1, m-k}^{i}=\sum_{j=1}^{k} \bar{p}_{j} x_{(j)}+\sum_{i=1}^{m-k} p_{i}^{*} a_{N-1, m-k}^{i} .
$$

In other words, if the decision-maker decides to assign $\bar{p}_{1}, \cdots, \bar{p}_{k}$ where

$$
\left\{\bar{p}_{1}, \cdots, \bar{p}_{k}\right\} \subset\left\{p_{1}, \cdots, p_{m}\right\}
$$


and $\bar{p}_{1} \geq \cdots \geq \bar{p}_{k}$, it is optimal to assign the $j$-th $p_{j}$ to the $j$-th $x_{(j)}$. It is, therefore,

$$
\begin{aligned}
& v_{N ; p_{1}, \cdots, p_{m}}\left(k ; x_{(1)}, \cdots, x_{(k)}\right) \\
= & \max _{\left\{\bar{p}_{1}, \cdots, \bar{p}_{k}\right\} \subset\left\{p_{1}, \cdots, p_{m}\right\}} \max _{\sigma \in \mathcal{S}_{k}}\left\{\sum_{j=1}^{k} \bar{p}_{\sigma(j)} x_{(j)}+v_{N-1 ; p_{1}^{*}, \cdots, p_{m-k}^{*}}\right\} \\
= & \max _{\left\{\bar{p}_{1}, \cdots, \bar{p}_{k}\right\} \subset\left\{p_{1}, \cdots, p_{m}\right\}}\left\{\sum_{j=1}^{k} \bar{p}_{j} x_{(j)}+\sum_{i=1}^{m-k} p_{i}^{*} a_{N-1, m-k}^{i}\right\} .
\end{aligned}
$$

From the above argument, this problem becomes how to assign $k$ of $\left\{x_{(i)}\right\}_{i=1, \cdots, k}$ and $m-k$ of $\left\{a_{N-1, m-k}^{i}\right\}_{i=1, \cdots, m-k}$ to $m$ of $\left\{p_{1}, \cdots, p_{m}\right\}$. In this case, $\left\{a_{N-1, m-k}^{i}\right\}_{i=1, \cdots, m-k}$ means the value that will be obtained in future decisions under the optimal policy.

For the union of two sets, $\left\{x_{(i)}\right\}_{i=1, \cdots, k}$ and $\left\{a_{N-1, m-k}^{i}\right\}_{i=1, \cdots, m-k}$, rearrange the order from largest to least as $\left\{b_{j}\right\}_{j=1,2, \cdots, m}$. Equation (3.6) is, therefore, equal to the following value.

$$
\max _{\sigma \in \mathcal{S}_{n}}\left\{\sum_{i=1}^{m} p_{i} b_{\sigma(i)}\right\} .
$$

On the other hand, since

$$
b_{1} \geq b_{2} \geq \cdots \geq b_{m}
$$

Lemma 1.1 yields

$$
\max _{\sigma \in \mathcal{S}_{n}}\left\{\sum_{i=1}^{m} p_{i} b_{\sigma(i)}\right\}=\sum_{i=1}^{m} p_{i} b_{i} .
$$

By these discussions we obtain the following facts. If $b_{j}=x_{(i)}$ where $j=1, \cdots, m$ and $i=1, \cdots, k$, then it is optimal to assign $x_{(i)}$ to the $j$-th $p_{j}$. On the other side, if $b_{j}=a_{N-1, m-k}^{i}$ where $j=1, \cdots, m$ and $i=1, \cdots, m-k$, then it is optimal not to assign the $j$-th $p_{j}$ at this period.

Proof of Proposition 3.2. We consider the value $a_{N, m}^{i}$ defined by Equations (2.12), (2.13) and (2.14). We will prove that the following facts. Under the optimal policy, this value is the expected amount by assigning to the $i$-th $p_{i}$ in the problem $P_{N ; p_{1}, \cdots, p_{m}}$ for $i(\leq m)$. This fact yields Equation (3.4) immediately. Similarly, $a_{N, m}^{i}(k)$ is the total expected amount by assigning to the $i$-th $p_{i}$ in the problem $P_{N ; p_{1}, \cdots, p_{m}}(k)$. We also employ the induction principle on $N$. It is obvious for $N=1$, and we assume these properties for any values less than $N-1$.

Initially we note the following fact.

$$
a_{N, m}^{i}(k)=U_{k}\left(a_{N-1, m-k}^{i}, a_{N-1, m-k}^{i-1} \mid 1, \infty\right) .
$$

Equation (2.7) yields

$$
\begin{aligned}
a_{N, m}^{1} & =U_{k}\left(a_{N-1, m-k}^{1}, \infty \mid 1, \infty\right) \\
& =\int_{0}^{a_{N-1, m-k}^{1}} a_{N-1, m-k}^{1} h_{k, 1}\left(x_{(1)}\right) f\left(x_{(1)}\right) d x_{(1)}
\end{aligned}
$$




$$
+\int_{a_{N-1, m-k}^{1}}^{\infty} x_{(1)} h_{k, 1}\left(x_{(1)}\right) f\left(x_{(1)}\right) d x_{(1)} .
$$

Under the optimal policy, we get the following facts. If $X_{(1)}>a_{N-1, m-k}^{1}$, it is optimal to assign a person with the largest value $p_{1}$. Otherwise, it is optimal not to assign $p_{1}$ to any job at this period. The second term of Equation (3.8) corresponds to the second case. Under the optimal policy, the induction assumption implies the following fact, i.e., during the rest of this problem $a_{N-1, m-k}^{1}$ is an expected amount by assigning to a man with the largest value. The first term of Equation (3.8), therefore, corresponds to the first case. By these discussions, this value is the total expected amount by assigning a person with the largest value $p_{1}$.

For the general case, Equation (2.6) yields the following equation.

$$
\begin{aligned}
a_{N, m}^{i}(k) & =U_{k}\left(a_{N-1, m-k}^{i}, a_{N-1, m-k}^{i-1} \mid 1, \infty\right) \\
& =\int_{0}^{a_{N-1, m-k}^{i}} a_{N-1, m-k}^{i} h_{k, 1}\left(x_{(1)}\right) f\left(x_{(1)}\right) d x_{(1)} \\
& +\int_{a_{N-1, m-k}^{i}}^{a_{N-1, m-k}^{i-1}} x_{(1)} h_{k, 1}\left(x_{(1)}\right) f\left(x_{(1)}\right) d x_{(1)} \\
& +\int_{a_{N-1, m-k}^{i-1}}^{y} U_{k}\left(a_{N-1, m-k}^{i-1}, a_{N-1, m-k}^{i-2} \mid 2, x_{(1)}\right) f\left(x_{(1)}\right) d x_{(1)}
\end{aligned}
$$

We will consider three terms of this equation when the decision-maker employs the optimal policy.

If $x_{(1)}<a_{N-1, m-k}^{i}$, then the decision-maker does not assign only $p_{i}$ but also any of $p_{1}, \cdots, p_{i-1}$. At the next period, the $p_{i}$ is still the $i$-th largest one. By the induction assumption, the expected amount by assigning to the $p_{i}$ is, therefore, equal to $a_{N-1, m-k}^{i}$. The first term of Equation (3.9) corresponds to this case.

If $a_{N-1, m-k}^{i} \leq x_{(1)}<a_{N-1, m-k}^{i-1}$, then the decision-maker assigns none of $p_{1}, \cdots$, $p_{i-1}$ and assigns the $p_{i}$ to a job with $x_{(1)}$. The second term of Equation (3.9) corresponds to this case.

Finally we consider the case that $a_{N-1, m-k}^{i-1} \leq x_{(1)}$. In this case, the decision-maker doesn't assign $p_{i}$ to $x_{(1)}$ but assigns one of the $p_{1}, \cdots, p_{i-1}$ to this job, since $a_{N-1, m-k}^{i-1} \leq$ $x_{(1)}$. Now we consider the following three cases according to the second largest value $x_{(2)}$. These three cases are (1) $x_{(2)}<a_{N-1, m-k}^{i-1}$, (2) $a_{N-1, m-k}^{i-1} \leq x_{(2)}<a_{N-1, m-k}^{i-2}$ and (3) $a_{N-1, m-k}^{i-2} \leq x_{(2)}$. As we considered above, these three cases correspond to the three cases for $x_{(1)}$. The function $U_{k}\left(a_{N-1, m-k}^{i-1}, a_{N-1, m-k}^{i-2} \mid 2, x_{(1)}\right)$ represents the sum of the expected amount for these three cases. The third term of Equation (3.9), therefore, corresponds to this case. This completes the proof.

REMARK 3.1. In Proposition 3.2, if we put $p_{1}=\cdots=p_{n}=1$ and $p_{n+1}=\cdots=$ $p_{m}=0$, then it is the same to Proposition 2.2. From this fact, the problem treated in this section is a generalization of an optimal selection problem considered before. 
REMaRK 3.2. Consider the case where $p_{1}=\cdots=p_{n}=1$ and $p_{n+1}=\cdots=p_{m}=0$. As we showed in the proof of Proposition $3.2, a_{N, m}^{n}$ is an expected amount obtained by the $n$-th $p_{n}$ in the problem $P_{N ; p_{1}, \cdots, p_{m}}$ for $n(\leq m)$. By this fact, if we add one more stop action to $P_{N, m, n-1}$, we will get an expected amount $a_{N, m}^{n}$ from this new action. For an optimal selection problem, $a_{N, m}^{n}$ is an increment of additional stop action to the problem $P_{N, m, n-1}$. It is, therefore, a value of the $n$-th action.

\section{Sequential Stochastic Assignment Problem with Unknown Number of Jobs}

In this section, we consider a sequential stochastic assignment problem for the unknown number of arriving jobs as treated in Section 2.3. The other condition is the same to the problem considered in 3 . When $N$ period remain, the number of jobs observed at the initial period is a random variable with a known probability distribution $p_{N}(k)\left(\sum_{k=1}^{\infty} k p_{N}(k)<\infty\right)$.

For this problem, there exists a sequence of threshold values which only depends on the density function of observations, which is similar to the one in Section 3. By these values, we get the optimal policy and the value obtainable under this policy.

Consider that the decision-maker observes iid non-negative random variables $\left\{X_{i}\right\}_{i=1, \cdots, \infty}$ during $N$ periods, and assign $\left\{p_{1}, \cdots, p_{n}\right\}$ to observations. We consider $\left(N ; p_{1}, \cdots, p_{n}\right)$ as a state variable for the problem in this section, and refer this problem as $Q_{N ; p_{1}, \cdots, p_{n}}$. For the problem $Q_{N ; p_{1}, \cdots, p_{n}}$, if the decision-maker can observe $k$ random variables, then let this sub-problem be $Q_{N ; p_{1}, \cdots, p_{n}}(k)$. Furthermore, when the $k$ observations of the random variables are $x_{(1)}, \cdots, x_{(k)}$, we state this sub-problem as $Q_{N ; p_{1}, \cdots, p_{n}}\left(k ; x_{(1)}, \cdots, x_{(k)}\right)$. Suppose $\left(x_{1}, \cdots, x_{k}\right)$ be an observation from the $k$ random variables $X_{1}, \cdots, X_{k}$. For these observations, rearrange the order as $x_{(1)}, \cdots, x_{(k)}$ where $x_{(1)} \geq \cdots \geq x_{(k)}$. This notation is similar to one used in the last section.

The objective of this problem is to maximize the total expected value by assigning the $n p$ 's to the $n$ selected values during a given time period $N$.

Let the total expected value obtainable under the optimal policy in $Q_{N ; p_{1}, \cdots, p_{n}}$, $Q_{N ; p_{1}, \cdots, p_{n}}(k)$ and $Q_{N ; p_{1}, \cdots, p_{n}}\left(k ; x_{(1)}, \cdots, x_{(k)}\right)$ be $v_{N ; p_{1}, \cdots, p_{n}}^{*}, \quad v_{N ; p_{1}, \cdots, p_{n}}^{*}(k)$ and $v_{N ; p_{1}, \cdots, p_{n}}^{*}\left(k ; x_{(1)}, \cdots, x_{(k)}\right)$, respectively. By the optimality principle, we get the following recursive equation (see Ross [13]).

$$
\begin{aligned}
& v_{N ; p_{1}, \cdots, p_{n}}^{*}=\sum_{k=0}^{\infty} v_{N ; p_{1}, \cdots, p_{n}}^{*}(k) p_{N}(k), \\
& v_{N ; p_{1}, \cdots, p_{n}}^{*}(k)=E\left[v_{N ; p_{1}, \cdots, p_{n}}^{*}\left(k ; X_{(1)}, \cdots, X_{(k)}\right)\right] \\
& v_{N ; p_{1}, \cdots, p_{n}}^{*}\left(k ; x_{(1)}, \cdots, x_{(k)}\right)= \\
& \max _{0 \leq l \leq k\left\{\bar{p}_{1}, \cdots, \bar{p}_{l}\right\} \subset\left\{p_{1}, \cdots, p_{n}\right\}} \max _{\sigma \in \mathcal{S}_{l}}\left\{\sum_{j=1}^{l} \bar{p}_{\sigma(j)} x_{(j)}+v_{\left.N-1 ; p_{1}^{*}, \cdots, p_{n-l}^{*}\right\}}^{*}\right\}
\end{aligned}
$$

The set $\left\{p_{1}^{*}, \cdots, p_{n-1}^{*}\right\}$ consists of remaining elements of $\left\{p_{1}, \cdots, p_{n}\right\}$ after eliminating the $l$ assigned elements of $\left\{\bar{p}_{1}, \cdots, \bar{p}_{l}\right\}$ at this period $\left(p_{1}^{*} \geq \cdots \geq p_{n-l}^{*}, \bar{p}_{1} \geq \cdots \geq \bar{p}_{l}\right)$. 
When the problem is $Q_{N ; p_{1}, \cdots, p_{n}}\left(k ; x_{(1)}, \cdots, x_{(k)}\right)$ and the decision-maker decides to select $i$ observations, he chooses $i$ largest values. We use this fact to obtain Equation (4.3).

Proposition 4.1. The optimal policy for sequential stochastic assignment problem $Q_{N ; p_{1}, \cdots, p_{n}}$ is represented as follows.

Consider the sub-problem that $Q_{N ; p_{1}, \cdots, p_{n}}\left(k ; x_{(1)}, \cdots, x_{(k)}\right)$. For the union two sets of $\left\{x_{(i)}\right\}_{i=1, \cdots, k}$ and $\left\{a_{N-1}^{i}\right\}_{i=1, \cdots, n}$, rearrange the order from largest to least as $\left\{b_{j}\right\}_{j=1,2, \cdots, n+k}$. If $b_{j}=x_{(i)}$ for $j=1, \cdots, n$ and $i=1, \cdots, k$, then it is optimal to assign the $j$-th $p_{j}$ to the observation $x_{(i)}$. If $b_{j}=a_{N-1}^{i}$ for $j=1, \cdots, n$ and $i=1, \cdots, n$, then it is optimal not to assign the $j$-th $p_{j}$ at this period. equations.

Proposition 4.2. The value $v_{N_{;} p_{1}, \cdots, p_{n}}^{*}$ and $v_{N ; p_{1}, \cdots, p_{n}}^{*}(k)$ satisfy the following

$$
\begin{aligned}
v_{N ; p_{1}, \cdots, p_{n}}^{*} & =\sum_{i=1}^{n} p_{i} a_{N}^{i}, \\
v_{N ; p_{1}, \cdots, p_{n}}^{*}(k) & =\sum_{i=1}^{n} p_{i} a_{N}^{i}(k) .
\end{aligned}
$$

In order to prove these properties, we employ the induction principle on $N$. We will prove Proposition 4.1 and Proposition 4.2 in the same time. First we prove these propositions for $N=1$. Then by assuming these properties for any value less than $N-1$, we will prove Proposition 4.1 and Proposition 4.2 for $N$.

When $N=1$, we have

$$
\max _{\sigma \in \mathcal{S}_{k}}\left\{\sum_{j=1}^{k \wedge n} \bar{p}_{\sigma(j)} x_{(j)}\right\} .
$$

As $k<n$, it is, therefore, optimal to assign the largest $k$ of $n p$ 's. In other words, it is optimal to assign $p_{1}, \cdots, p_{k}$ to the $k$ observations. Lemma 1.1 yields Proposition 4.1. Since

$$
\begin{aligned}
v_{1 ; p_{1}, \cdots, p_{n}}^{*} & =\sum_{i=1}^{n} p_{i} \sum_{k=0}^{\infty} p_{1}^{k} \mathrm{E}\left[X_{(i)}^{k}\right] \\
& =\sum_{i=1}^{n} p_{i} a_{1}^{i},
\end{aligned}
$$

we get Proposition 4.1. (See Remark 2.3) Next we assume these propositions for any value less than $N-1$. We obtain the proof of Proposition 4.1 by a method similar to one used in Proposition 3.2, and omit the proof here.

Proof of Proposition 4.2. First we consider the following equation where $\left\{\bar{p}_{1}, \cdots, \bar{p}_{k}\right\} \subset\left\{p_{1}, \cdots, p_{n}\right\}$.

$$
\max _{\sigma \in \mathcal{S}_{l}}\left\{\sum_{j=1}^{1} \bar{p}_{\sigma(j)} x_{(j)}+v_{N-1 ; p_{1}^{*}, \cdots, p_{n-1}^{*}}^{*}\right\} .
$$


The induction assumption yields

$$
v_{N-1 ; p_{1}^{*}, \cdots, p_{n-1}^{*}}^{*}=\sum_{i=1}^{n-l} p_{i}^{*} a_{N-1}^{i} .
$$

This implies, therefore,

$$
\begin{aligned}
\max _{\sigma \in \mathcal{S}_{l}}\left\{\sum_{j=1}^{l} \bar{p}_{\sigma(j)} x_{(j)}+v_{N-1 ; p_{1}^{*}, \cdots, p_{n-l}^{*}}^{*}\right\} & =\max _{\sigma \in \mathcal{S}_{l}}\left\{\sum_{j=1}^{l} \bar{p}_{\sigma(j)} x_{(j)}+\sum_{i=1}^{n-l} p_{i}^{*} a_{N-1}^{i}\right\} \\
& =\max _{\sigma \in \mathcal{S}_{l}}\left\{\sum_{j=1}^{l} \bar{p}_{\sigma(j)} x_{(j)}\right\}+\sum_{i=1}^{n-l} p_{i}^{*} a_{N-1}^{i} .
\end{aligned}
$$

Lemma 1.1 and Equation (4.6) yield

$$
\max _{\sigma \in \mathcal{S}_{l}}\left\{\sum_{j=1}^{l} \bar{p}_{\sigma(j)} x_{(j)}\right\}+\sum_{i=1}^{n-l} p_{i}^{*} a_{N-1}^{i}=\sum_{j=1}^{l} \bar{p}_{j} x_{(j)}+\sum_{i=1}^{n-l} p_{i}^{*} a_{N-1}^{i} .
$$

From this equation, if the decision-maker decides to assign

$$
\left\{\vec{p}_{1}, \cdots, \bar{p}_{l}\right\} \subset\left\{p_{1}, \cdots, p_{n}\right\}
$$

at this period, it is optimal to assign $j$-th largest $\bar{p}_{j}$ to the $j$-th $x_{(j)}$ where $1 \leq j \leq l$. It becomes, therefore, the following equality.

$$
\begin{aligned}
& v_{N ; p_{1}, \cdots, p_{n}}^{*}\left(k ; x_{(1)}, \cdots, x_{(k)}\right) \\
& \quad=\max _{1 \leq l \leq k} \max _{\left\{\bar{p}_{1}, \cdots, \bar{p}_{l}\right\} \subset\left\{p_{1}, \cdots, p_{n}\right\}} \max _{\sigma \in \mathcal{S}_{l}}\left\{\sum_{j=1}^{l} \bar{p}_{\sigma(j)} x_{(j)}+v_{N-1 ; p_{1}^{*}, \cdots, p_{n-l}^{*}}^{*}\right\} \\
& \quad=\max _{1 \leq l \leq k} \max _{\left\{\bar{p}_{1}, \cdots, \bar{p}_{l}\right\} \subset\left\{p_{1}, \cdots, p_{n}\right\}}\left\{\sum_{j=1}^{l} \bar{p}_{j} x_{(j)}+\sum_{i=1}^{n-l} p_{i}^{*} a_{N-1}^{i}\right\} .
\end{aligned}
$$

Next we consider the following equation.

$$
\max _{\left\{\bar{p}_{1}, \cdots, \bar{p}_{l}\right\} \subset\left\{p_{1}, \cdots, p_{n}\right\}}\left\{\sum_{j=1}^{l} \bar{p}_{j} x_{(j)}+\sum_{i=1}^{n-l} p_{i}^{*} a_{N-1}^{i}\right\} .
$$

Consider the union of two sets $\left\{x_{(i)}\right\}_{i=1, \cdots, l}$ and $\left\{a_{N-1}^{i}\right\}_{i=1, \cdots, n-l}$, and denote this set as $B_{l}$, i.e.,

$$
B_{l}=\left\{x_{(1)}, \cdots, x_{(l)}, a_{N-1}^{1}, \cdots, a_{N-1}^{n-l}\right\}
$$

This problem is how to assign the element of $B_{l}$ to $\left\{p_{1}, \cdots, p_{n}\right\}$. For $B_{l}$, rearrange the order from largest to least and let this rearranged set be $\left\{b_{j}^{l}\right\}_{j=1,2, \cdots, n}$. By simple calculation, we get, therefore,

$$
\max _{\left\{\bar{p}_{1}, \cdots, \bar{p}_{l}\right\} \subset\left\{p_{1}, \cdots, p_{n}\right\}}\left\{\sum_{j=1}^{l} \bar{p}_{j} x_{(j)}+\sum_{i=1}^{n-l} p_{i}^{*} a_{N-1}^{i}\right\}=\max _{\sigma \in \mathcal{S}_{n}}\left\{\sum_{i=1}^{n} p_{i} b_{\sigma(i)}^{l}\right\} .
$$


Since

$$
b_{1}^{l} \geq b_{2}^{l} \geq \cdots \geq b_{n}^{l}
$$

Lemma 1.1 yields

$$
\max _{\sigma \in \mathcal{S}_{l}}\left\{\sum_{i=1}^{n} p_{i} b_{\sigma(i)}^{l}\right\}=\sum_{i=1}^{n} p_{i} b_{i}^{l} .
$$

We consider the policy to choose $l$ largest observation from $k$. When $b_{j}^{l}=x_{(i)}$ for $j=1, \cdots, n$ and $i=1, \cdots, k$, then it is optimal to assign $j$-th $p_{j}$ to $x_{(i)}$. If $b_{j}=a_{N-1}^{i}$ for $j=1, \cdots, n$ and $i=1, \cdots, n-k$, it is optimal not assign $j$-th $p_{j}$ at this period.

Next define the value of $l$ for the optimal policy. Equation (4.7) yields

$$
v_{N ; p_{1}, \cdots, p_{n}}^{*}\left(k ; x_{(1)}, \cdots, x_{(k)}\right)=\max _{1 \leq l \leq k}\left\{\sum_{i=1}^{n} p_{i} b_{i}^{l}\right\} .
$$

Put

$$
\xi_{l}=\sum_{i=1}^{n} p_{i} b_{i}^{l} .
$$

On the other hand, consider the union of two sets of $\left\{x_{(1)}, \cdots, x_{(k)}\right\}$ and $\left\{a_{N-1}^{1}, \cdots, a_{N-1}^{n}\right\}$. For this set, rearrange the order from largest to least. From this rearranged set, select the $n$ largest elements, and let the number of $x_{(i)}$ 's contained in this set be $l^{*}$. This set is $\left\{x_{(1)}, \cdots, x_{\left(l^{*}\right)}, a_{N-1}^{1}, \cdots, a_{N-l^{*}}^{n}\right\}$, and $x_{\left(l^{*}+1\right)} \leq a_{N-1}^{n-l^{*}}$. This fact yields $x_{\left(l^{*}\right)} \geq a_{N-1}^{n-l^{*}} \geq x_{\left(l^{*}+1\right)}$ Then we get

$$
\xi_{l^{*}}=\sum_{i=1}^{n} p_{i} b_{i}^{l^{*}}
$$

If $l^{*} \leq l$, we put $b_{j}=x_{\left(l^{*}\right)}$ for some $j$ where $1 \leq j \leq n$. Then we have the following equality.

$$
\begin{aligned}
\xi_{l^{*}}-\xi_{l} & =\sum_{i=1}^{n} p_{i} b_{i}^{l^{*}}-\sum_{i=1}^{n} p_{i} b_{i}^{l} \\
& =\sum_{i=j}^{n} p_{i} b_{i}^{l^{*}}-\sum_{i=j}^{n} p_{i} b_{i}^{l} \\
& =\sum_{i=j}^{n} p_{i} a_{N-1}^{i-l^{*}}-\sum_{i=j}^{n} p_{i} b_{i}^{l} \\
& =\sum_{i=j}^{n} p_{i}\left\{a_{N-1}^{i-l^{*}}-b_{i}^{l}\right\} .
\end{aligned}
$$

Note the fact that $B_{l}$ is a union of two sets $\left\{x_{(i)}\right\}_{i=1, \cdots, l}$ and $\left\{a_{N-1}^{i}\right\}_{i=1, \cdots, n-l}$. For $B_{l}$, rearrange the order from greatest to least, and compare two sets $B_{l}$ and $B_{l^{*}}$. Then the $j$ largest elements of these two sets are the same. Eliminating these $j$ values, we consider 
the remaining elements of these two sets. Denote the remainder of these two sets as $\bar{B}_{l}$ and $\bar{B}_{l^{*}}$. Then we have

$$
\bar{B}_{l^{*}}=\left\{a_{N-1}^{j-l^{*}}, a_{N-1}^{j+1-l^{*}}, \cdots, a_{N-1}^{n-l^{*}}\right\}
$$

where $a_{N-1}^{j-l^{*}} \geq a_{N-1}^{j+1-l^{*}} \geq \cdots \geq a_{N-1}^{n-l^{*}}$. On the other hand, put $l=l^{*}+\bar{l}+1$ where $\bar{l} \geq 0$. Then we also obtain

$$
\bar{B}_{l}=\left\{a_{N-1}^{j-l^{*}}, a_{N-1}^{j+1-l^{*}}, \cdots, a_{N-1}^{n-l^{*}-1}, x_{(l-\bar{l})}, \cdots, x_{(l-1)}, x_{(l)}\right\}
$$

where $a_{N-1}^{j-l^{*}} \geq a_{N-1}^{j+1-l^{*}} \geq \cdots \geq a_{N-1}^{n-l^{*}-1} \geq x_{(l-\bar{l})} \geq \cdots \geq x_{(l-1)} \geq x_{(l)}$. Since $l-\vec{l}=l^{*}+1$, we note the following inequality.

$$
x_{(l)} \leq \cdots \leq x_{\left(l^{*}+1\right)}=x_{(l-\bar{l})} \leq a_{N-1}^{n-l^{*}} .
$$

By comparing two sets $B_{l}$ and $B_{l^{*}}$, Equation (4.10) yields

$$
\xi_{l *}-\xi_{l}=\sum_{j=l-\bar{l}}^{l}\left\{a_{N-1}^{n-l^{*}-(l-j)}-x_{(j)}\right\} \geq 0 .
$$

We get, therefore,

$$
\xi_{l^{*}} \geq \xi_{l}
$$

This completes the proof.

REMark 4.1. In Proposition 4.2, put $p_{1}=\cdots=p_{n}=1$ and $p_{n+1}=\cdots=p_{m}=0$. Then this proposition is the aforementioned to Proposition 3.2. The sequential stochastic assignment problem treated here is a generalization of an optimal selection problem with unknown number of jobs in 2.3 .

REMARK 4.2. As in the last remark, when $p_{1}=\cdots=p_{n}=1$ and $p_{n+1}=\cdots=$ $p_{m}=0$, we have that $Q_{N, i}=P_{1, \cdots, 1,0, \cdots, 0}$. We showed the following fact in the proof of Proposition 4.2. The value $a_{N}^{i}$ is a total expected amount obtained by the $i$-th $p_{i}$ in the problem $P_{N ; p_{1}, \cdots, p_{n}}$ for $i(\leq m)$. By these facts, if we add one more stop action to the problem $Q_{N, i-1}$, we will get an expected value $a_{N}^{i}$ from this new action. For an optimal selection problem, $a_{N}^{i}$ is an increment of additional stop action to the problem $Q_{N, i-1}$. It is, therefore, a value of the $i$-th action.

\section{References}

[1] S. C. Albright, Optimal Sequential Assignments with Random Arriving Time, Management Science, vol. 21, pp. 60-67, 1974.

[2] M. H. DeGroot, Optimal Statistical Decisions, McGraw-Hill, New York, New York, 1970.

[ 3 ] C. Derman, G. J. Lieberman and S. M. Ross, A Sequential Stochastic Assignment Problem, Management Science, vol. 18, pp. 349-355, 1972. 
[4] G. H. Hardy, J. E. Littlewood and G. Polya, Inequality, Cambridge University Press, 1934.

[5] T. Nakai, Optimal Assignment for a Random Sequence with an Unknown Parameter, Journal of Information E Optimization Sciences, vol. 1, pp. 129-138 1980.

[6] T. Nakai, Sequential Stochastic Assignment Problem with Rejection, Journal of Information ES Optimization Sciences, vol. 2, 169-181, 1981.

[7] T. Nakai, A Time Sequential Game Related to the Sequential Assignment Problem, Journal of the Operations Research Society of Japan, vol. 25, pp. 129-138, 1982.

[8] T. Nakai, Game of the Sequential Assignment for the Randomly Arriving Jobs, Reports of Statistical Applications Research, Union of Japanese Scientists and Engineers, vol. 29, 1-6, 1986.

[ 9 ] T. Nakai, Optimal Assignment for a Random Sequence with an Unknown Number of Jobs, Journal of the Operations Research Society of Japan, vol. 28, pp. 179-194, 1985.

[10] T. Nakai, An Optimal Selection Problem with a Random Number of Applicants per Period, Operations Research, vol. 34, pp. 478-485, 1986.

[11] T. Nakai, A Sequential Stochastic Assignment Problem in a Partially Observable Markov Chain, Mathematics of Operations Research, vol. 11, pp. 230-240, 1986.

[12] T. Nakai, A Sequential Stochastic Assignment Problem in a Stationary Markov Chain, Mathematica Japonica, vol. 31, 741-757, 1986.

[13] S. M. Ross, Applied Probability with Optimization Applications, Holden-Day, San Francisco, California, 1970.

Received May 28, 1993

Revised September 16, 1993

Communicated by S. Iwamoto 